\title{
Clay Catalyzed Reactions of Indole and its Methyl Derivatives with $\alpha, \beta$-unsaturated Carbonyl Compounds
}

\author{
Teslima Dasbasia,* and Meysun I. Abdullah ${ }^{\mathrm{b}}$ \\ ${ }^{a}$ Bozok University, Faculty of Science \& Arts, Department of Chemistry, 66200, Yozgat,Turkey \\ ${ }^{\mathrm{b}}$ Nigde University, Faculty of Science \& Arts, Department of Chemistry, 51200, Nigde,Turkey
}

RECEIVED APRIL 22, 2013; REVISED FEBRUARY 28, 2014; ACCEPTED MARCH 18, 2014

\begin{abstract}
Electrophilic substituons reactions of indole and 1-methylindole with methyl propiolate in the presence of K-10 montmorillonite were obtained the formation of the corresponding methyl 3,3bis(indolyl)propanoates. The reaction of 1,3-dimethylindole with methyl propiolate was given methyl 3,3bis(1,3-dimethyl- $1 H$-indol-2-yl)propanoate, methyl 1,5-dimethyl- $1 H$-benzo[b]azepine-3-carboxylate and methyl 3,3,3-tris(1,3-dimethyl-1H-indol-2-yl)propanoate. The reaction of 1,3-dimethylindole with 2cyclopentenone was yielded a typical addition product, similarly the reaction of indole and 1-methylindole with 2-cyclopentenone were concluded the expected addition products only.
\end{abstract}

Keywords: Michael addition, bisindolylester, trisindolylester, benzoazepine, K-10 montmorillonite

\section{INTRODUCTION}

Indole and its derivatives are components of drugs founded in many pharmaceutical compounds ${ }^{1-4}$ and are crucial building blocks for biologically active compounds. $^{5,6}$ The Michael addition of indoles to $\alpha, \beta$ unsaturated carbonyl compounds are an useful reaction for medicinal chemistry applications. Bis and trisindolyl compounds have recieved a considerable attention on account of their biological activity ${ }^{7-9}$ and growth inhibitory activity on tumor cells. ${ }^{10-13}$ Trisindolyl amines are reported to be important intermediates for the development of new drugs with potential ironchelating abilities. ${ }^{14}$ However, for years, many synthetic methods for the preparation of the biologically important, diindolyl ${ }^{15-22}$ and trisindolylalkanes, ${ }^{23-27}$ have been reported and most of these procedures either in strong acidic conditions, ${ }^{28,29}$ expensive reagents and catalysts involved ${ }^{30-35}$ or they were carried out under dry conditions using microwaves ${ }^{36,37}$ and ultrasound accelerated methods. ${ }^{38}$

Environmentally, Benign chemical processes using less hazardous catalysts has become a primary goal in synthetic organic chemistry. In this work, the reactions of indole, 1-methylindole and 1,3-dimethylindole with methyl propiolate and 2-cyclopentenone in dichloromethane under mild conditions using K-10 montmorillonite as catalyst is described. The reactions of indole and methyl-substituted indoles with $\alpha, \beta$ - unsaturated carbonyl compounds with an initial attack at the preferred 3-position of the indoles were followed by rearrangement to the 2-position. This type of rearrangement has been previously reported by Jackson et al. $^{39-41}$

Treatment of indole(1) and 1-methylindole(2) with methyl propiolate in dichloromethane in the presence K10 montmorillonite, occurred just at that position to give methyl 3,3-di(1H-indol-2-yl)propanoate(4) and 3,3-bis(1-methyl-1H-indol-2-yl)propanoate(5) (Scheme $1)$. The $\mathrm{C}^{3}$ atom in the indole molecule is the most active in electrophilic substitution processes. ${ }^{23,31}$ In the molecule of 1,3-dimethylindole(3), because the 3position is occupied by methyl group, from the addition reaction of 1,3-dimethylindole(3) to methyl propiolate in dichloromethane and K-10 Montmorllonite catalyst were obtained three different products in one pot;

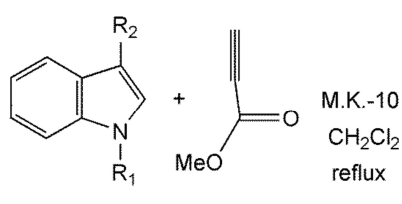

(1): $R_{1}, R_{2}=H$

(2): $\mathrm{R}_{1}=\mathrm{CH}_{3}, \mathrm{R}_{2}=\mathrm{H}$

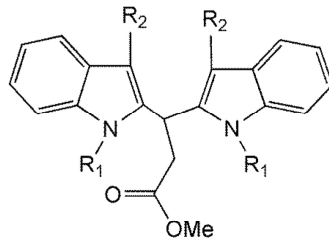

(4): $R_{1}, R_{2}=H$

(5): $\mathrm{R}_{1}=\mathrm{CH}_{3}, \mathrm{R}_{2}=\mathrm{H}$
Scheme 1. The reaction of indole and 1-methylindole with methyl propiolate.

\footnotetext{
* Author to whom correspondence should be addressed. (E-mail: teslima.dasbasi@bozok.edu.tr \& teslimadasbasi@hotmail.com)
} 
<smiles>C=CC(=O)OCC(=O)OC</smiles><smiles>CC(=O)CC(c1c(C)c2ccccc2n1C)c1c(C)c2ccccc2n1C</smiles>

(6) $\mathrm{OMe}$<smiles></smiles>

(7)

and<smiles>COC(=O)CC(c1c(C)c2ccccc2n1C)(c1c(C)c2ccccc2n1C)c1c(C)c2ccccc2n1C</smiles>

(8)

Scheme 2. The reaction of 1,3-dimethylindole with methyl propiolate.

methyl 3,3-bis(1,3-dimethyl-1H-indol-2-yl)propanoate(6), methyl 1,5-dimethyl-1H-benzo[b]azepin-3carboxylate(7) and the methyl 3,3,3-tris(1,3-dimethyl$1 \mathrm{H}$-indol-2-yl)propanoate(8) respectively (Scheme 2). Substituted benzoazepines possess a broad spectrum of biological activities. ${ }^{42}$ They are of moderate size giving rise to their potential as ligands for receptors and offer semi-restricted conformational flexibility allowing considerable scope for selective binding with a range of functional groups. ${ }^{14}$ Benzoazepine type compounds were previously synthesized from the 2-methoxyindole and dimethyl pyrroles in $1966 .^{43}$ It is worth mentioning that in previous work, ${ }^{44}$ we have reported the synthesis of dimethyl 2-(2-methyl-1H-methylindol-3-yl)maleate and dimethyl 2-methyl-1H-1-benzazopine-3,4-dicarboxylate from the reaction of 2-metylindole with dimethyl acetylenedicarboxylate. From the reaction 1,3-dimethylindole and dimethyl acetylenedicarboxylate were isolated dimethyl 1,5-dimethyl-1H-1-benzoazepine-3,4dicarboxylate. In 1,3-dimethylindole with methylpropiolate reaction as a successful example of the ring expansion was obtained methyl 1,5-dimethyl- $1 H$ benzo[b]azepin-3-carboxylate(7).<smiles>[R]c1cn([R8])c2ccccc12</smiles>

(1): $R_{1}, R_{2}: H$

(2): $R_{1}: M e, R_{2}: H$

(3): $R_{1}: M e, R_{2}: M e$

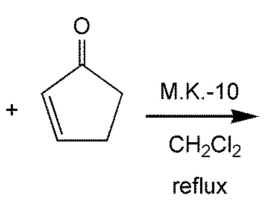

reflux

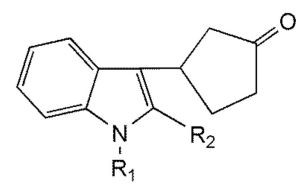

(9): $\mathbf{R}_{1}, \mathbf{R}_{2}: \mathrm{H}$

(10): $R_{1}: M e, R_{2}: H$ (11): $R_{1}: M e, R_{2}: M e$
Scheme 3. The reaction of indole, 1-methylindole and 1,3dimethylindole with 2-cyclopentenone.
Indole(1), 1-methylindole(2) and 1,3-Dimethylindole(3) was also reacted with 2-cyclopentenone under the same conditions and in this reaction, it is yielded only Michael addition products (Scheme 3 ). We have found that Montmorillonite smoothly catalyzes in these reactions leading to two $\mathrm{C}-\mathrm{C}$ bonds and thus affording the desired products in one pot.

\section{EXPERIMENTAL}

\section{Material}

All chemicals were purchased from Merck, Fluka and Sigma-Aldrich and Montmorillonite K-10 clay was purchased from Fluka AG, Switzerland. TLC was carried out on aluminum sheets precoated with silica gel $60 \mathrm{~F}_{254}$ (Merck), and the spots were visualized with UV light $(\lambda=254 \mathrm{~nm})$. Column chromatography was conducted on silica gel $60(40-63 \mu \mathrm{m})$. The melting points were determined on an Electrotermal A 9100 melting point apparatus. The NMR spectra were recorded on a Bruker DPX-400 spectrometer. Chemical shifts are reported in parts per million relative to $\mathrm{CHCl}_{3}\left({ }^{1} \mathrm{H}: \delta\right.$ $=7.27), \mathrm{CDCl}_{3}\left({ }^{13} \mathrm{C}: \delta=77.0 \mathrm{ppm}\right)$ and $\mathrm{CCl}_{4}\left({ }^{13} \mathrm{C}: \delta=\right.$ $96.4 \mathrm{ppm})$. The IR spectra were measured in $\mathrm{KBr}$ on a Jasco FTIR 300E spectrometer. The mass spectra were run on an LC/MS, AGILENT 1100 MSD system. The elemental compositions were determined using a LECO CHNS-932 analyzer.

\section{Synthesis of Methyl 3,3-di(1H-indol-2-yl)propano- ate(4)}

$8 \mathrm{mmol}$ of indole(1) and $4 \mathrm{mmol}$ methyl propiolate was dissolved in $50 \mathrm{ml}$ dichloromethane and K-10 catalyst 
was added. The mixture was refluxed for 6 hours and the progress of the reaction was monitored by TLC. After the removal of the solvents under reduced pressure, the crude product was purified by column chromatography on flash silica gel using (ethylacetate / cyclohexane, 3:7, v/v) followed by recrystallization from ethylacetate / cyclohexane to give (e.g. $0.93 \mathrm{~g}, 73 \%$ ) red crystals,. m.p. $79-80 .{ }^{\circ} \mathrm{C} ;{ }^{1} \mathrm{H}$ NMR $\left(400 \mathrm{MHz}, \mathrm{CDCl}_{3}\right)$ $\delta /$ ppm: 7.9 (2H, s, N-H), 7.55 (2H, d, $J=6.8 \mathrm{~Hz}, \mathrm{H}-7)$, $7.22(2 \mathrm{H}, \mathrm{d}, J=8.1 \mathrm{~Hz}, \mathrm{H}-4), 7.16(2 \mathrm{H}, \mathrm{t}, J=8 \mathrm{~Hz}, \mathrm{H}-5)$, $7.03(2 \mathrm{H}, \mathrm{t}, J=10 \mathrm{~Hz}, \mathrm{H}-6), 6.83(2 \mathrm{H}, \mathrm{s}, \mathrm{H}-2), 5.1(1 \mathrm{H}$, t, $J=7.5 \mathrm{~Hz}, \mathrm{CH}), 3.55\left(3 \mathrm{H}, \mathrm{s}, \mathrm{OCH}_{3}\right), 3.28(2 \mathrm{H}, \mathrm{d}, J$ $\left.=9.7 \mathrm{~Hz}, \mathrm{CH}_{2}\right) .{ }^{13} \mathrm{C} \mathrm{NMR}\left(400 \mathrm{MHz}, \mathrm{CDCl}_{3}\right) \delta / \mathrm{ppm}$ : $173.2(1 \mathrm{C}, \mathrm{C}=\mathrm{O}), 136.6$ (2C, C-8), 126.6 (2C, C-9), 121.9 (2C, C-2), 121.8 (2C, C-5), 119.4 (2C, C-6), 119.2 (2C, C-4), 118.6 (2C, C-3), 111.2 (2C, C-7), 51.6 $\left(1 \mathrm{C}, \mathrm{OCH}_{3}\right), 41.0(1 \mathrm{C}, \mathrm{CH}), 30.8\left(1 \mathrm{C}, \mathrm{CH}_{2}\right) ; \mathrm{MS} m / z$ : $318\left(\mathrm{M}^{+}, 3 \%\right), 282\left(\mathrm{M}^{+}-34,17 \%\right), 276\left(\mathrm{M}^{+}-42,100\right.$ $\%), 216\left(\mathrm{M}^{+}-132,14 \%\right)$. Anal. Calcd. mass fractions of elements, $w / \%$, for $\mathrm{C}_{20} \mathrm{H}_{18} \mathrm{~N}_{2} \mathrm{O}_{2}\left(M_{\mathrm{r}}=318.37\right)$ are: $\mathrm{C}$ 75.45, H 5.70, N 8.80; found: C 75.38, H 5.64, N 8.66.

\section{Synthesis of Methyl 3,3-bis(1-methyl-1H-indol-2- yl)propanoate(5)}

Into a solution of 1-methylindole $(2)(8 \mathrm{mmol})$ and methyl propiolate $(4 \mathrm{mmol})$ in $50 \mathrm{~mL}$ dichloromethane, the K-10 catalyst was added. The mixture was refluxed for 6 hours and the reaction was followed by TLC. After completion, the catalyst was filtered off and washed with dichloromethane. The reaction products were separated by flash chromatography (chloroform / petroleum ether, 9:1, v/v) followed by recrystallization from chloroform / petroleum ether to give e.g. $0.86 \mathrm{~g}$ (62\%) white crystals, m.p. $122-123{ }^{\circ} \mathrm{C} .{ }^{1} \mathrm{H}$ NMR (400 MHz, $\mathrm{CDCl}_{3}$ ) $\delta$ /ppm: 7.5 (d, $\left.J=8 \mathrm{~Hz}, 2 \mathrm{H}, \mathrm{H}-7\right), 7.3$ (d, $J=6.7$, 2H, Hz, H-4), 7.2 (t, $J=10 \mathrm{~Hz}, 2 \mathrm{H}, \mathrm{H}-5), 7.0$ (t, $J=10$ Hz, 2H, H-6), 6.8 (s, 2H, H-2), 5.1 (t, J=7.5 Hz, 1H, $\mathrm{CH}), 3.7\left(\mathrm{~s}, 6 \mathrm{H}, \mathrm{N}-\mathrm{CH}_{3}\right), 3.5\left(\mathrm{~s}, 3 \mathrm{H}, \mathrm{OCH}_{3}\right), 3.1(\mathrm{~d}, J=$ $\left.7.5 \mathrm{~Hz}, 2 \mathrm{H}, \mathrm{CH}_{2}\right) ;{ }^{13} \mathrm{C} \mathrm{NMR}\left(400 \mathrm{MHz}, \mathrm{CDCl}_{3}\right) \delta / \mathrm{ppm}$ : $172.9(1 \mathrm{C}, \mathrm{C}=\mathrm{O})$, 137.3 (2C, C-8), 128.6 (2C, C-9), 127.0 (2C, C-2), 126.3 (2C, C-5), 121.4 (2C, C-6), 118.8 (2C, C-4), 117.4 (2C, C-3), 109.1 (2C, C-7), 51.5 $\left(1 \mathrm{C}, \mathrm{OCH}_{3}\right), 41.3(1 \mathrm{C}, \mathrm{CH}), 32.6\left(2 \mathrm{C}, \mathrm{N}-\mathrm{CH}_{3}\right), 30.6$ (1C, $\left.\mathrm{CH}_{2}\right)$; $\mathrm{MS} \mathrm{m} / z: 347\left(\mathrm{M}^{+}, 4 \%\right), 285\left(\mathrm{M}^{+}-61,38 \%\right)$ $202\left(\mathrm{M}^{+}-144,68 \%\right), 170\left(\mathrm{M}^{+}-176,88 \%\right), 160\left(\mathrm{M}^{+}-186\right.$, $100 \%$ ). Anal. Calcd. mass fractions of elements, $w / \%$, for $\mathrm{C}_{22} \mathrm{H}_{22} \mathrm{~N}_{2} \mathrm{O}_{2}\left(M_{r}=346.42\right)$ are: $\mathrm{C} 76.28, \mathrm{H} 6.40, \mathrm{~N}$ 8.09; found: C 76.14, H 6.35, N 8.24.

\section{The Reaction of 1,3-dimethylindole with Methyl Propiolate}

1,3-dimethylindole(3) (12 mmol) was dissolved in 50 $\mathrm{mL}$ dichloromethane and $4 \mathrm{mmol}$ of methyl propiolate was added. After the addition of $2 \mathrm{~g}$ of K-10 Mont- morillonite clay, the mixture was refluxed for 16 hours and the progress of the reaction was monitored by TLC. After completion, the reaction mixture was filtered under vacuum and the solvent was evaporated off to give an oily product. This mixture was separated by flash chromatography using chloroform / petroleum ether, $(4: 1, v / v)$ to give the following three products; methyl 3,3-bis(1,3-dimethyl-1H-indol-2-yl) propanoate(6), methyl 1,5-dimethyl-1H-benzo[ $b]$ azepine-3carboxylate(7) and methyl 3,3,3-tris(1,3-dimethyl-1Hindol-2-yl) propanoate( 8 ) respectively.

\section{Methyl 3,3-bis(1,3-dimethyl-1H-indol-2-yl)propano- ate(6)}

Pale yellow crystals, recrystallized from chloroform / petroleum ether, e.g. $0.48 \mathrm{~g}(23 \%)$, m.p. $87-88{ }^{\circ} \mathrm{C} ;{ }^{1} \mathrm{H}$ NMR (400 MHz, $\mathrm{CDCl}_{3}$ ) $\delta / \mathrm{ppm}: 7.52$ (d, $J=5 \mathrm{~Hz}, 2 \mathrm{H}$, $\mathrm{H}-7), 7.2$ (d, $J=7.3 \mathrm{~Hz}, 2 \mathrm{H}, \mathrm{H}-4), 7.1$ (t, $J=7 \mathrm{~Hz}, 2 \mathrm{H}, \mathrm{H}-$ 5), 6.9 (t, $J=7 \mathrm{~Hz}, 2 \mathrm{H}, \mathrm{H}-6), 5.1(\mathrm{t}, J=7.5 \mathrm{~Hz}, 1 \mathrm{H}, \mathrm{CH})$, $3.7\left(\mathrm{~s}, 6 \mathrm{H}, \mathrm{N}-\mathrm{CH}_{3}\right), 3.5\left(\mathrm{~s}, 3 \mathrm{H}, \mathrm{OCH}_{3}\right), 3.1(\mathrm{~d}, J=7.6$ $\left.\mathrm{Hz}, 2 \mathrm{H}, \mathrm{CH}_{2}\right), 2.1\left(\mathrm{~s}, 6 \mathrm{H}, \mathrm{CH}_{3}\right) ;{ }^{13} \mathrm{C}$ NMR $(400 \mathrm{MHz}$, $\left.\mathrm{CDCl}_{3}\right) \delta / \mathrm{ppm}: 173(1 \mathrm{C}, \mathrm{C}=\mathrm{O}), 137(2 \mathrm{C}, \mathrm{C}-2), 127$ (2C, C-8), 126 (2C, C-9), 121 (2C, C-5), 119 (2C, C-6), 118 (2C, C-4), 117 (2C, C-7), 109 (2C, C-3), 51 (1C, $\left.\mathrm{OCH}_{3}\right), 41\left(1 \mathrm{C}, \mathrm{CH}_{2}\right), 32\left(2 \mathrm{C}, \mathrm{N}-\mathrm{CH}_{3}\right), 30(1 \mathrm{C}, \mathrm{CH}), 29$ $\left(2 \mathrm{C}, \mathrm{CH}_{3}\right)$; $\mathrm{MS} m / z: 375\left(\mathrm{M}^{+}, 5 \%\right) 345\left(\mathrm{M}^{+}-29,8 \%\right)$, $275\left(\mathrm{M}^{+}-99,4 \%\right), 216\left(\mathrm{M}^{+}-158,38 \%\right), 184\left(\mathrm{M}^{+}-190\right.$, $100 \%$ ). Anal. Calcd. mass fractions of elements, $w / \%$, for $\mathrm{C}_{24} \mathrm{H}_{26} \mathrm{~N}_{2} \mathrm{O}_{2}\left(M_{r}=374.48\right)$ are: $\mathrm{C} 76.98, \mathrm{H} 7.00, \mathrm{~N}$ 7.48; found: C 76.84, H 7.10, N 7.36.

\section{Methyl 1,5-dimethyl-1H-benzo[b]azepine-3-carboxy- late(7)}

Yellow crystals, recrystallized from chloroform / petroleum ether, e.g. $0.35 \mathrm{~g}$ (17\%), m.p. $106-107{ }^{\circ} \mathrm{C}$; ${ }^{1} \mathrm{H}$ NMR (400 MHz, $\mathrm{CDCl}_{3}$ ) $\delta / \mathrm{ppm}: 7.85$ (s, 1H, H-2), 7.75 (s, 1H, H-4), 7.5 (d, $J=6$ Hz, 1H, H-6), 7.20-7.15 (m, 1H, H-8), 7.05-6.95 (m, 1H, H-7), 6.25 (d, $J=6 \mathrm{~Hz}$, 2H, H-9), 3.7 (s, 3H, $\left.\mathrm{OCH}_{3}\right), 2.4\left(\mathrm{~s}, 3 \mathrm{H}, \mathrm{N}-\mathrm{CH}_{3}\right), 1.45$ (s, 3H, benzo[b]azepin-5- $\left.\mathrm{CH}_{3}\right) ;{ }^{13} \mathrm{C} \mathrm{NMR}(400 \mathrm{MHz}$, $\left.\mathrm{CDCl}_{3}\right) \delta /$ ppm: $168(1 \mathrm{C}, \mathrm{C}=\mathrm{O}), 138(1 \mathrm{C}, \mathrm{C}-10), 132$ (1C, C-2), 127 (2C, C-8), 123 (1C, C-6), 119 (2C, C11), 116 (1C, C-4), 115 (1C, C-7), 108 (2C, C-9), 102 $(2 \mathrm{C}, \mathrm{C}-3), 51\left(1 \mathrm{C}, \mathrm{OCH}_{3}\right), 29.7\left(1 \mathrm{C}, \mathrm{N}-\mathrm{CH}_{3}\right), 10(1 \mathrm{C}$, $\left.\mathrm{CH}_{3}\right) ; \mathrm{MS} m / z: 229\left(\mathrm{M}^{+}, 11 \%\right), 160\left(\mathrm{M}^{+}-69,100 \%\right)$, $149\left(\mathrm{M}^{+}-80,18 \%\right), 113\left(\mathrm{M}^{+}-116,18 \%\right)$. Anal. Calcd. mass fractions of elements, $w / \%$, for $\mathrm{C}_{14} \mathrm{H}_{15} \mathrm{NO}_{2}\left(M_{r}=\right.$ 229.27) are: C 73.34, H 6.59, N 6.11; found: C 73.20, H 6.53, N 6.26 .

\section{Methyl 3,3,3-tris(1,3-dimethyl-1 $H$-indol-2-yl)propa- noate(8)}

Dark green crystals, recrystallized from chloroform / petroleum ether, e.g. $0.79 \mathrm{~g}(38 \%)$ m.p. $119-120{ }^{\circ} \mathrm{C}$; 
${ }^{1} \mathrm{H}$ NMR $\left(400 \mathrm{MHz}, \mathrm{CDCl}_{3}\right) \delta / \mathrm{ppm}: 7.9(\mathrm{~d}, J=6 \mathrm{~Hz}, 3 \mathrm{H}$, H-7), 7.7-7.5 (m, 3H, H-4), 7.3-7.2 (m, 3H, H-5), 7.2-7.0 (m, 3H, H-6), 3.85 (d, $\left.J=3.3 \mathrm{~Hz}, 9 \mathrm{H}, \mathrm{N}-\mathrm{CH}_{3}\right)$, $3.65\left(\mathrm{~d}, J=3.3 \mathrm{~Hz}, 3 \mathrm{H}, \mathrm{OCH}_{3}\right), 3.1\left(\mathrm{~s}, 2 \mathrm{H}, \mathrm{CH}_{2}\right), 2.5(\mathrm{~s}$, $\left.3 \mathrm{H}, \mathrm{CH}_{3}\right), 2.2\left(\mathrm{~s}, 6 \mathrm{H}, \mathrm{CH}_{3}\right) ;{ }^{13} \mathrm{C}$ NMR $(400 \mathrm{MHz}$, $\left.\mathrm{CDCl}_{3}\right) \delta / \mathrm{ppm}: 167.0(1 \mathrm{C}, \mathrm{C}=\mathrm{O}), 138.2(3 \mathrm{C}, \mathrm{C}-2), 132.6$ (d, $J=1.6 \mathrm{~Hz}, 2 \mathrm{C}, \mathrm{C}-8), 131.3(1 \mathrm{C}, \mathrm{C}-8), 128.1$ (3C, C9), 124.2 (3C, C-5), 122.5 (3C, C-6), 119.5 (3C, C-4), 117.3 (2C, C-7), 116.3 (1C, C-7), $112.8(1 \mathrm{C}, \mathrm{C}-3)$, $109.2(\mathrm{~d}, J=0.5 \mathrm{~Hz}, 2 \mathrm{C}, \mathrm{C}-3), 51.6\left(2 \mathrm{C},-\mathrm{OCH}_{3},-\mathrm{CH}_{2}\right)$, $30.7\left(4 \mathrm{C}, \mathrm{N}-\mathrm{CH}_{3},-\mathrm{C}\right), 10.3\left(3 \mathrm{C},-\mathrm{CH}_{3}\right)$. M S m/z : 517 $\left(\mathrm{M}^{+}, 4 \%\right), 487\left(\mathrm{M}^{+}-30,6 \%\right), 459\left(\mathrm{M}^{+}-58,10 \%\right), 230$ $\left(\mathrm{M}^{+}-287,100 \%\right), 198\left(\mathrm{M}^{+}-319,91 \%\right)$. Anal. Calcd. mass fractions of elements, $w / \%$, for $\mathrm{C}_{34} \mathrm{H}_{35} \mathrm{~N}_{3} \mathrm{O}_{2}\left(M_{\mathrm{r}}=\right.$ 517.66) are: $\mathrm{C} 78.89, \mathrm{H} 6.81, \mathrm{~N} 8.12$; found: $\mathrm{C} 79.01, \mathrm{H}$ $6.86, \mathrm{~N} 7.98$.

\section{Synthesis of 3-(1H-indol-3-yl)cyclopentanone(9)}

$6 \mathrm{mmol}$ of indole was dissolved in $40 \mathrm{ml}$ dichloromethane followed by the addition of $3 \mathrm{mmol} 2-$ cyclopentenone and $2 \mathrm{~g}$ of the catalyst Montmorillonite. The mixture was refluxed for $4 \mathrm{~h}$ and the reaction was observed by TLC. After the removal of the solvents under reduced pressure, the crude product was purified by column chromatography on flash silica gel using (ethylacetate/cyclohexane, 1:1, v/v). Dark green crystals, e.g. $0.62 \mathrm{~g}(65 \%)$ m.p. $61-63{ }^{\circ} \mathrm{C} ;{ }^{1} \mathrm{H}$ NMR $(400$ $\left.\mathrm{MHz}, \mathrm{CDCl}_{3}\right) \delta / \mathrm{ppm}: 8.1(\mathrm{~s}, 1 \mathrm{H}, \mathrm{N}-\mathrm{H}), 7.6(\mathrm{~d}, 1 \mathrm{H}, J=8$ $\mathrm{Hz}, \mathrm{H}-7), 7.4(\mathrm{~d}, 1 \mathrm{H}, J=7 \mathrm{~Hz}, \mathrm{H}-4), 7.3-7.2(\mathrm{~m}, 1 \mathrm{H}, \mathrm{H}-$ 5), 7.2-7.1 (m, 1H, H-6), 7.0 (s, 1H, H-2), 3.8-3.6 (m, $1 \mathrm{H}$, cyclopentanon-H-3), 2.8-2.7 (m, $2 \mathrm{H}$, cyclopentanon-H-2), 2.6-1.8 (m, 4H, cyclopentanon- $\mathrm{H}-4$, cyclopentanon-H-5); ${ }^{13} \mathrm{C}$ NMR $\left(400 \mathrm{MHz}, \mathrm{CDCl}_{3}\right)$ $\delta / \mathrm{ppm}: 219.2(1 \mathrm{C}, \mathrm{C}=\mathrm{O}), 137.3(1 \mathrm{C}, \mathrm{C}-8), 126.6(1 \mathrm{C}, \mathrm{C}-$ 9), 122.3 (1C, C-2), 119.9 (1C, C-5), 119.5 (1C, C-6), 119.0 (1C, C-4), 118.7 (1C, C-3), 111.3 (1C, C-7), 45.2 (1C, cyclopentanon-C-3), 38.1 (1C, cyclopentanon-C2), 33.7 (1C, cyclopentanon-C-5), 29.9 (1C, cyclopentanon-C-4); MS $m / z: 200.2\left(\mathrm{M}^{+}, 24 \%\right), 149.1\left(\mathrm{M}^{+}-50\right.$, 28\%), $118.1\left(\mathrm{M}^{+}-81,37 \%\right), 97.1\left(\mathrm{M}^{+}-102,100 \%\right), 83.2$ $\left(\mathrm{M}^{+}-102\right.$, 21\%). Anal. Calcd. mass fractions of elements, $w / \%, \mathrm{C}_{13} \mathrm{H}_{13} \mathrm{NO}:\left(M_{\mathrm{r}}=199.25\right)$ are: $\mathrm{C} 78.36$, H 6.58, N 7.03; found: C 78.24, H 6.64, N 7.15.

\section{Synthesis of 3-(1-methyl-1H-indol-3-yl)cyclopenta- none(10)}

$4 \mathrm{~g}$ of Montmorillonite was added in to a mixture of 8 mmol of 1-methylindole and $4 \mathrm{mmol}$ of 2cyclopentenone in $40 \mathrm{~mL}$ dicloromethane. The mixture was refluxed for $4 \mathrm{~h}$ and the reaction product was flash chromatographed using ethylacetate / petroleum ether $(3: 7, v / v)$. Brown crystals, e.g. 0.84 g, (61 \%) m.p 91-92 ${ }^{\circ} \mathrm{C}$; ${ }^{1} \mathrm{H}$ NMR $\left(400 \mathrm{MHz}, \mathrm{CDCl}_{3}\right) \delta / \mathrm{ppm}$ : $7.6(\mathrm{~d}, 1 \mathrm{H}, J=8$ Hz, H-7), 7.3-7.2 (m, 2H, H-4, H-5), 7.1-7.0 (m, 1H,
H-6), 6.7 (s, 1H, H-2), 3.7 (s, 3H, N- $\mathrm{CH}_{3}$ ), 2.7-2.6 (m, $1 \mathrm{H}$, cyclopentanon-H-3), 2.4-2.2 (m, 4H, cyclopentanon-H-2, cyclopentanon-H-4), 2.1-2.0 (m, 2H, cyclopentanon- $\mathrm{H}-5) ;{ }^{13} \mathrm{C}$ NMR $\left(400 \mathrm{MHz}, \mathrm{CDCl}_{3}\right)$ $\delta / \mathrm{ppm}: 219.2(1 \mathrm{C}, \mathrm{C}=\mathrm{O}), 137.4(1 \mathrm{C}, \mathrm{C}-8), 127.0(1 \mathrm{C}, \mathrm{C}-$ 9), 124.0 (1C, C-2), 121.9 (1C, C5), 119.1 (1C, C-6), 118.9 (1C, C-4), 117.1 (1C, C-7), 109.4 (1C, C-3), 45.4 (1C, cyclopentanon-C-3), $38.1\left(1 \mathrm{C}, \mathrm{N}-\mathrm{CH}_{3}\right), 33.7(1 \mathrm{C}$, cyclopetanon-C-2), 32.6 (1C, cyclopentanon-C-5), 30.8 (1C, cyclopentanon-C-4); MS m/z : $213\left(\mathrm{M}^{+}, 17 \%\right)$, $200.2\left(\mathrm{M}^{+}-13,5 \%\right), 152.1\left(\mathrm{M}^{+}-61,3 \%\right), 113.2\left(\mathrm{M}^{+}-\right.$ $100,100 \%)$. Anal. Calcd. for $\mathrm{C}_{14} \mathrm{H}_{15} \mathrm{NO}:\left(M_{\mathrm{r}}=213.12\right)$ are: C 78.84, H 7.09, N 6.57; found: C 78.98, H 7.04, N 6.45 .

\section{Synthesis of 3-(1,2-dimethyl-1H-indol-2-yl)cyclopen- $\operatorname{tanon}(11)$}

$3 \mathrm{~g}$ of Montmorillonite was added in to a mixture of 8 mmol 1,3-methylindole and $4 \mathrm{mmol} 2$-cyclopentenon in $30 \mathrm{~mL}$ dichloromethane and refluxed for $4 \mathrm{~h}$. The resulting mixture was chromatographed using chloroform / petroleum ether $(3: 2, v / v)$ and a brownish gum like product was obtained. (e.g. $1.11 \mathrm{~g}, 74$ \%), m.p 138-139 ${ }^{\circ} \mathrm{C}$; ${ }^{1} \mathrm{H}$ NMR $\left(400 \mathrm{MHz}, \mathrm{CDCl}_{3}\right) \delta / \mathrm{ppm}: 7.5(\mathrm{~d}, 1 \mathrm{H}$, $J=7.8 \mathrm{~Hz}, \mathrm{H}-7), 7.3-7.1$ (m, 1H, H-4, H-5), 7.1-7.0 (m, $1 \mathrm{H}, \mathrm{H}-6), 3.7\left(\mathrm{~s}, 3 \mathrm{H}, \mathrm{N}-\mathrm{CH}_{3}\right), 2.8-2.6(\mathrm{~m}, 1 \mathrm{H}$, cyclopentanon-H-3), 2.6-2.5 (m, 2H, cyclopentanon-H2), 2.5-2.3 (m, 7H, cyclopentanon-H-4, cyclopentanon$\left.\mathrm{H}-5, \mathrm{CH}_{3}\right) ;{ }^{13} \mathrm{C}$ NMR $\left(400 \mathrm{MHz}, \mathrm{CDCl}_{3}\right) \delta / \mathrm{ppm}: 217.6$ $(1 \mathrm{C}, \mathrm{C}=\mathrm{O}), 136.6(1 \mathrm{C}, \mathrm{C}-2), 135.4(1 \mathrm{C}, \mathrm{C}-8), 128.7$ (1C, C-9), 121.4 (1C, C-5), 119.0 (1C,C-6), 118.0 (1C, C-4), 111.6 (1C, C-7), 108.7 (1C, C-3), 43.3 (1C, cyclopentanon-C-2), 38.8 (1C, cyclopentanon-C-5), $34.2\left(1 \mathrm{C}, \mathrm{N}-\mathrm{CH}_{3}\right), 30.2$ (1C, cyclopentanone-C-4), 29.6 (1C, cyclopentanon-C-3), $9.7\left(1 \mathrm{C}, \mathrm{CH}_{3}\right) ; \mathrm{MS} \mathrm{m} / \mathrm{z}$ : $228.2\left(\mathrm{M}^{+}, 50 \%\right), 214.2\left(\mathrm{M}^{+}-13,8 \%\right), 146.2\left(\mathrm{M}^{+}-81\right.$, $21 \%), 97\left(\mathrm{M}^{+}-130,100 \%\right)$. Anal. Calcd. for $\mathrm{C}_{15} \mathrm{H}_{17} \mathrm{NO}$ : $\left(M_{r}=227.3\right)$ are: $\mathrm{C} 79.26, \mathrm{H} 7.54, \mathrm{~N} 6.16$; found: $\mathrm{C}$ 79.14, H 7.54, N 6.02 .

\section{RESULTS AND DISCUSSION}

In this study, the bis, trisindolyl and benzo[b]azepine type compounds have been obtained directly from the reaction of 1,3-dimethylindole(3) with methyl propiolate rather than reacting indoles with aldehydes or ketons. ${ }^{23-27}$ The reaction of 1,3-dimethylindole(3) with methyl propiolate (Scheme 2) proceeds with an initial attack at the preferred 3-position of the 1,3dimethylindole. The intra-molecular arrangement followed by the ring expansion gave the cyclic product, methyl 1,5-dimethyl- $1 H$-benzo[ $b]$ azepine-3-carboxylate(7). This reaction is a successful example of the ring expansion under the action of activated acetylenes. ${ }^{43-45}$ 
Substituted benzoazepines possess a broad spectrum of biological activities, as the benzoazepine ring is a major fragment of a series of alkaloids. ${ }^{33,47}$ However, when the resonate was further attacked by a second molecule of 1,3-dimethyl indole, the reaction was also proceeded with the attack at the 3-position followed by rearrangement to the 2-position to give the methyl 3,3-bis(1,3dimethyl-1H-indol-2-yl)propanoate(6) at higher yield. Jackson et al have previously reported similar type of rearrangement. ${ }^{41}$ The methyl 3,3,3-tris(1,3-dimethyl$1 H$-indol-2-yl)propano-ate(8) was obtained at lower yield. The reaction of indole and 1-methylindole with methyl propiolate gave only diindolyl products and no cyclization or trisindolyl products were observed (Scheme 1).

However, the reaction of indole, 1-methylindole and 1,3-dimethylindole with 2-cyclopentenone were isolated addition products only and no cyclization or trisindolyl products were obtained (Scheme 3).

\section{CONCLUSION}

Michael addition of indoles to the $\alpha, \beta$-unsaturated carbonyl compounds is an important approach to carboncarbon bond forming reactions in organic synthesis. ${ }^{42-47}$ The reaction is a typical Michael type 1,4-addition or conjugate addition of resonance-stabilized carbanions of the methyl propiolate. The reaction of indole and 1methylindole with methyl propiolate could afford addition products and bisindolyl products only (Scheme 1). However, Montmorillonite smoothly catalyzes these reactions leading to two $\mathrm{C}-\mathrm{C}$ bonds. The use of clay in these reactions was found to be very attractive, because of its environmental compatibility. Many protic acids and Lewis acids that are used in these reactions are sometimes deactivated. When Lewis acids are used, the excess acid can liberate as harmful mixtures to the eco system.

Acknowledgements. The authors would like to thank the Research fund of the University of Nigde for the financial support.

\section{REFERENCES}

1. X. Gu, W. Zhuo, and B. Jiang, Bioorg. Med. Chem. Lett. 9 (1999) 569-572.

2. K. Singh, J. Singh, P. K. Deb, and H. Singh, Heterocycles 55 (1999) 12873-12880.

3. L. Chacun-Lefevne, V. Benteteau, B. Joseph, and J. Y. Merour, Tetrahedron 58 (2002) 10181-10188.

4. S. Hibino and T. Choshi, Nat. Prod. Rep. 19 (2002) 148-180.

5. D. Komander, G. S. Kular, A. W. Schütterkopf, M. Deak, K. R. C. Prakash, J. Bain, M. Elliott, M. Garrido-Franco, A. P. Kozikowski, D. R. Alessi, and D. M. F. Aalten, Structure 12 (2004) 215-226.

6. G. W. Gribble, J. Chem. Soc., Perkin Trans. 17 (2000)
1045-1075.

7. F. Lingens and W. Goebel, Biochim. Biophys. Acta, Gen. Subj. 148 (1967) 70-83.

8. H. Alinezhad, H. A. Haghighi, and F. Salehian, Chinese Chem. Lett. 21 (2010) 183-186.

9. C. Aubry, A. J. Wilson, D. Emmerson, E. Murphy, Y. Y. Chan, M. P. Dickens, M. D. Garcia, P. R. Jenkins, S. Mahale, and B. Chaudhuri, Bioorgan. Med. Chem. 17 (2009) 6073-6084.

10. X. Chang, J. C. Tou, C. Hong, H. A. Kim, J. E. Riby, G. L. Firestone, and L. F. Bjeldanes, Carcinogenesis (2005) 771-778.

11. M. York, M. Abdelrahim, S. Chintharlapalli, S. D. Lucerok, and S. Safe, Clin. Cancer Res. 13 (2007) 6743-6752.

12. T. Inamoto, S. Papineni, S. Chintharlapalli, S. D. Cho, S. Safe, and A. M. Kamat, Mol. Cancer Ther. 7 (2008) 3825-3833.

13. N. Ichite, M. B. Chougule, T. Jackson, S. V. Fulzele, S. Safe, and M. Singh, Clin. Cancer Res. 15 (2009) 543-552.

14. R. B. Sears, R. A. Carpenter, and C. R. Whitlock, Molecules 10 (2005) 488-491.

15. J. S. Yadav, V.S. Reddy, B. Padmavam, and M. K. Gupta, Tetrahedron Lett. 45 (2004) 7577-7579.

16. S. Kumar, V. Kumar, and S. S. Chimni, Tetrahedron Lett. 44 (2003) 2101-2104.

17. B.P. Bandgar and K.A. Shaikh Tetrahedron Lett. 44 (2003) 1959-1961.

18. C.J. Magesh, R. Nagarajan, M. Karthik, and P. Perumal, Appl. Catal. A: Gen. 266 (2004) 1-10.

19. M. Chakrabarty, R. Basak, N. Ghosha, and Y. Harigaya, Tetrahedron 60 (2004) 1941-1949.

20. P. K. Bowyer, D. S. Black, and D. C. Craig, Tetrahedron 61 (2005) 10781-10792.

21. M. A. Zolfigol, P. Salehi, M. Shiri, and Z. Tanbakouchian, Catal. Commun. 8 (2007) 173-178.

22. C. W. Kuo, C. C. Wang, H. L. Fang, B. R.Raju, V. Kavala, P. M. Habib, and C. F. Yao, Molecules 14 (2009) 3952-3963.

23. M. Chacrabarty and S. Sarkar, Tetrahedron Lett. 43 (2002) 1351-1353.

24. J. C. Magesh, R. Nagarajan, and P. T. Perumal, Chem. Lett. 33 (2004) 288-289.

25. K. Tabatabaeian, M. Mamaghani, N. O. Mahmoodi, and A. Khorshidi, J. Mol. Catal. A-Chem. 270 (2007) 112-116.

26. D. Nematollahi and S. Dehdashtian, Tetrahedron Lett. 49 (2008) 645-649.

27. M. Shiri, M. A. Zolfigol, H. G. Kruger, and Z. Tanbakouchian, Chem. Rev. 110 (2010) 2250-2293.

28. M. Agnusdei, M. Bandini, A. Melloni, and A. Umani-Ronchi, J. Org Chem. 68 (2003) 7126-7129.

29. A. V. Reddy, K. Ravinder, Goud P. Krishnaiah, T. V. Raju, and Y. Venkateswarlu, Tetrahedron Lett. 44 (2003) 6257-6260.

30. S. Y. Wang, S. J. Ji, and T. P. Loh, Synlett. (2003) 2377-2379.

31. P. Shanmugam, P. Rajasingh Tetrahedron 60 (2004) 9283-9295.

32. J.S. Yadav, B.V.S Reddy, B. Eeshwaraiah, and M. Srinivas Tetrahedron 60 (2004) 1767-1771

33. J.S. Yadav, B.V.S.Reddy, and G. Satheesh Tetrahedron Lett. 45 (2004) 3673-3676.

34. G. Bartoli, M. Bartolacci, M. Boxco, G. Foglia, A. Giuliani, E. Marcantoni, L. Sambri, and E. Torregiani, J. Org Chem. 68 (2003) 4594-4597.

35. A. Dhakshinamoorthy, Synlett. 19 (2005) 3014-3015.

36. M. Karthik, A. K. Tripathi, N. M. Gupta, M. Palanichamy, and V. Murugesan, Catal. Commun. 5 (2004) 371-375.

37. J. D. Pranab and D. Jupitara Tetrahedron Lett. 53 (2012) 4718 4720 .

38. Z. L. Shen, S. J. Ji, S.Y. Wang, and X. F. Zeng, Tetrahedron (2005) 10552-10558.

39. A. H. Jackson, N. Prasitpan, P. V. R. Shannon, and A. C. Tinker, J. Chem. Soc., Perkin Trans. 1 (1987) 2543-2551. 
40. A. H. Jackson and P. P. Lynch, J. Chem. Soc., Perkin Trans. 2 (1987) 1215-1219.

41. M. I. Abdullah, A. H. Jackson, P. P. Lynch, and K. A. F. Record, Heterocycles 30 (1990) 317-320.

42. V. Varlamov, D. G. Grudinin, A. I. Chernyshev, A. N. Levov, I. Y. Lobanov, R. S. Borisov, and F. I. Zubkov, Russ. Chem. B 53 (2004) 1711-1716.

43. H. Plieninger and D. Wild, Chem Ber. (1966) 3070-3075.
44. O. Sarioz and M. I. Abdullah, Russ. J. Org. Chem. 42 (2006) 879-882.

45. R. L. Garnick, S. B. Levery, and P. W. Le Quesne, J. Org. Chem. 43 (1978) 1226-1229.

46. P. D. Davis, D. C. Neckers, and J. R. Blount, J. Org. Chem. 45 (1980) 462-471.

47. V. Kouznetsov, A. Palma, and C. Ewert, Curr. Org. Chem. 5 (2001) 519-551. 